\title{
Dopamine receptor D4 gene (DRD4) is associated with gazing toward humans in domestic dogs (Canis familiaris)
}

\author{
Yusuke Hori ${ }^{1^{*}}$, Hisayo $\mathrm{Kishi}^{2}$, Miho Inoue-Murayama ${ }^{3}$, Kazuo Fujita $^{1}$ \\ ${ }^{1}$ Department of Psychology, Graduate School of Letters, Kyoto University, Kyoto, Japan; \\ *Corresponding Author: hori.yuusuke.74z@st.kyoto-u.ac.jp \\ ${ }^{2}$ Otani Ladies Clinic, Kobe, Japan \\ ${ }^{3}$ Wildlife Research Center, Kyoto University, Kyoto, Japan
}

Received 30 November 2012; revised 4 January 2013; accepted 14 January 2013

\begin{abstract}
Dogs show high social communicative ability in interactions with humans. We investigated the association between dogs' social communicative behavior and the polymorphisms of a gene related to a neurotransmitter. We used an "unsolvable task", in which an experimenter put a food reward into a container and closed it firmly so that dogs could not remove the reward. Human-directed gazing, possibly to request help, is a characteristic behavioral trait of dogs in such situations. The association between owner-directed gazing behavior in the unsolvable task and polymorphisms of three regions (exon1, exon3, intron2) in the dopamine receptor D4 gene (DRD4) was analyzed. We found that the genotype of DRD4 intron2 was significantly associated with the dogs' gazing behavior. Dogs carrying shorter allele $(P)$ looked at their owner more frequently, for longer, and earlier than dogs carrying longer allele $(Q)$. This result suggests that polymorphism in DRD4 intron2 may affect social communication and cognition in dogs.
\end{abstract}

Keywords: Dogs; Dopamine Receptor D4 Gene; Human-Directed Gazing; Social Behavior

\section{INTRODUCTION}

Dogs (Canis familiaris) are characterized by their high social communicative ability in interactions with humans. For example, they are very sensitive to human gestures (e.g. pointing) and attentional states [1,2]. Furthermore, dogs outperform their ancestors, wolves [3]. Such abilities are sometimes called "human-like social skills" [4]. In particular, dogs engage in prominent human-directed gazing. They do this, possibly to request help, in difficult situations in which wolves do not show the behavior [5]. This dissociation suggests that human-directed gazing maybe have taken on increasing importance in dogs during domestication from their ancestral wolverine condition. However, the genetics behind social cognition and behavior of dogs remains unclear.

Recent research has suggested that genes related to neurotransmitters or hormones contribute to individual variability of behavioral traits in many species [6]. The dopamine receptor $\mathrm{D} 4$ gene $(D R D 4)$ is a strong potential candidate gene in this respect. Polymorphism in $D R D 4$ exon3 is reportedly associated with a novelty-seeking trait in humans $[7,8]$. In canine $D R D 4$, polymorphisms are found in 3 regions (exon1, exon3, intron2). For exon1 and intron2, these polymorphisms are based on insertion/deletion of short unit $[9,10]$. For exon3, the polymorphism is based on a variable number of tandem repeat (VNTR) [11]. Previous studies showed that these polymorphisms are associated with several behavioral traits. Exon3 is associated with aggressiveness and activity/impulsivity [12], and intron2 is reportedly associated with social impulsivity [13].

Appropriate measurement of behavioral traits is essential for studying the effects of genes on behavior. Previous studies in domesticated animals have often relied on questionnaires given to caretakers [14]. However, behavioral testing may allow more objective behavioral evaluation. In the present study, we presented dogs with an "unsolvable task" that they could not solve by themselves. We analyzed the association between dogs' human-directed gazing behavior during the unsolvable task and the genotypes of three regions in $D R D 4$.

\section{MATERIAL AND METHODS}

\subsection{Animals}

Fifty-five pet dogs (25 males and 30 females) partici- 
pated; 20 (8 males and 12 females) lived in Berlin, Germany and 35 (17 males and 18 females) lived in Kinki region of Japan. The mean age of dogs was 4.7 years (SD $=2.9$ ). Various breeds were included; 43 were pure breeds and 12 were mongrels (see Table 1). The present study was approved by the ethical committee at the Wildlife Research Center, Kyoto University (no. WRC-2012EC001).

Table 1. The results of genotyping for each breed.

\begin{tabular}{|c|c|c|c|c|c|c|c|c|}
\hline \multirow{2}{*}{ Breed } & \multirow{2}{*}{$N$} & \multicolumn{2}{|c|}{ Exon1 } & \multicolumn{2}{|c|}{ Exon3 } & \multicolumn{3}{|c|}{ Intron2 } \\
\hline & & $S-$ & $S+$ & $435-$ & $435+$ & $P / P$ & $P / Q$ & $Q / Q$ \\
\hline Airedale Terrier & 2 & 2 & 0 & 1 & 1 & 2 & 0 & 0 \\
\hline American Pit Bull Terrier & 1 & 0 & 1 & 0 & 1 & 0 & 0 & 1 \\
\hline Basset Hound & 1 & 1 & 0 & 1 & 0 & 0 & 0 & 1 \\
\hline Border Collie & 1 & 0 & 1 & 0 & 1 & 0 & 1 & 0 \\
\hline Cavalier King Charles Spaniel & 1 & 1 & 0 & 0 & 1 & 1 & 0 & 0 \\
\hline Chihuahua & 2 & 0 & 2 & 0 & 2 & 1 & 1 & 0 \\
\hline Dachshund & 1 & 1 & 0 & 1 & 0 & 0 & 1 & 0 \\
\hline English Springer Spaniel & 1 & 0 & 1 & 0 & 1 & 0 & 1 & 0 \\
\hline Golden Retriever & 1 & 0 & 1 & 1 & 0 & 0 & 0 & 1 \\
\hline Hungarian Vizla & 3 & 1 & 2 & 3 & 0 & 0 & 1 & 2 \\
\hline Jack Russell terrier & 2 & 1 & 1 & 1 & 1 & 1 & 1 & 0 \\
\hline Japanese Spitz & 2 & 2 & 0 & 2 & 0 & 1 & 0 & 1 \\
\hline Labrador Retriever & 3 & 1 & 2 & 1 & 2 & 0 & 1 & 2 \\
\hline Miniature Dachshund & 1 & 1 & 0 & 0 & 1 & 0 & 1 & 0 \\
\hline Miniature Schnauzer & 2 & 2 & 0 & 2 & 0 & 0 & 1 & 1 \\
\hline Mongrel & 12 & 5 & 7 & 10 & 2 & 0 & 3 & 9 \\
\hline Pomeranian & 2 & 1 & 1 & 0 & 2 & 1 & 1 & 0 \\
\hline Rottweiler & 1 & 1 & 0 & 0 & 1 & 1 & 0 & 0 \\
\hline Saluki & 1 & 0 & 1 & 1 & 0 & 0 & 0 & 1 \\
\hline Scottish Deerhound & 1 & 1 & 0 & 0 & 1 & 1 & 0 & 0 \\
\hline Sealyham Terrier & 1 & 1 & 0 & 1 & 0 & 1 & 0 & 0 \\
\hline Shetland Sheepdog & 1 & 0 & 1 & 0 & 1 & 0 & 1 & 0 \\
\hline Japanese Shiba & 1 & 1 & 0 & 1 & 0 & 0 & 1 & 0 \\
\hline Siberian Husky & 1 & 0 & 1 & 1 & 0 & 0 & 0 & 1 \\
\hline Toy Poodle & 4 & 2 & 2 & 4 & 0 & 0 & 1 & 3 \\
\hline Welsh Corgi Pembroke & 1 & 1 & 0 & 1 & 0 & 1 & 0 & 0 \\
\hline Welsh Springer Spaniel & 1 & 0 & 1 & 1 & 0 & 0 & 0 & 1 \\
\hline West Highland White Terrier & 1 & 1 & 0 & 1 & 0 & 0 & 1 & 0 \\
\hline Whippet & 2 & 2 & 0 & 0 & 2 & 2 & 0 & 0 \\
\hline Wire-Haired Fox Terrier & 1 & 0 & 1 & 1 & 0 & 0 & 0 & 1 \\
\hline Total & 55 & 29 & 26 & 35 & 20 & 13 & 17 & 25 \\
\hline
\end{tabular}




\subsection{Behavioral Test}

The behavioral test was conducted in a room unfamiliar to dogs. One dog, one dog's owner, and the experimenter, an unfamiliar male, were in the room. The owner and the experimenter stood or sat opposite each other. The experimenter first put a piece of the dog's favorite food into a transparent plastic container measuring $12 \times$ $12 \times 5 \mathrm{~cm}$, and encouraged the dog to eat the reward from there ("familiarization trial"). After two familiarization trials, the experimenter put the food into the container and placed a lid on it so that dogs could take the reward easily by touching the box ("easy trial”). After one easy trial, the experimenter again put food into the box and closed the lid firmly so that dogs could not open it to get the food ("difficult trial”). We videotaped dogs' behavior during the difficult trial and coded gazing toward the owner. We coded the frequency, duration, and latency of the gazing behavior. The duration and latency were coded frame by frame (30 frame/sec). The coder was ignorant of the genotype of dogs. To test the reliability of coding a second coder (also ignorant of the genotype) coded $20 \%$ of the videos $(N=11)$. The agreement between coders was high ( $r=0.91$ for frequency, 0.98 for duration, and 0.97 for latency).

\subsection{Genetic Analysis}

After the behavioral test buccal cells or hairs were collected from the dogs. Genomic DNA were extracted from buccal swabs using QIAamp blood and tissue kit (QIAGEN, Valencia, CA, USA), or from hairs using InstaGene $^{\text {TM }}$ Matrix (Bio-Rad laboratories, Shinagawa, Tokyo, Japan). Three regions in DRD4 (exon1, exon3, and intron2) were amplified by polymerase chain reaction (PCR) and genotyped. PCR condition and primers followed [9] for exon1 and exon3, and [10] for intron2. PCR products were electrophoresed using the Applied Biosystems 3130xl Genetic Analyzer (Applied Biosystems, Foster City, CA, USA) and the sizes of PCR products were measured using the Gene Mapper software package (Applied Biosystems).

\subsection{Statistical Analysis}

We used generalized linear models (GLM) to analyze the association between behavioral indices and genotype. We analyzed three behavioral indices (frequency, duration, and latency) separately. We applied Poisson distribution to frequency and normal distribution to duration and latency. Sex, age, and genotypes of three regions (exon1, exon3 and intron2) were added to the model as explanatory variables. The effect of country (Japan or Germany) was not included in the model because none of the means of the three indices differed significantly between the two countries (all $p$-values $>0.05$ ). The significance of the effect of each variable was tested by the likelihood ratio test using chi-square statistics (type III test). All analyses were conducted using the software $\mathrm{R}$ 2.14.0 (R Development Core Team).

\section{RESULTS}

\subsection{Genotyping}

The result of genotyping is summarized in Table 1. For exon1, two alleles (short allele: $S$, long allele: $L$ ) were found. For exon3, five alleles (435, 447a, 447b, 498, 549 ) were found. For intron2, two alleles (short allele: $P$, long allele: $Q$ ) were found.

For exon1 and exon3, dogs were categorized based on the existence or no-existence of shorter allele (exon1: $S$, exon3: 435) because the number of individuals was not sufficient to categorize based on the genotype. For intron2, dogs were categorized based on their genotype $(P / P, P / Q$, and $Q / Q)$.

\subsection{Association between the Genotype and the Behavioral Indices}

Table 2 shows the result of likelihood ratio test by GLM.

Table 2. The results of likelihood ratio tests for each variable. Chi-square statistic and $p$-value are shown. Significant $p$-values are shown in bold.

\begin{tabular}{|c|c|c|c|c|c|c|}
\hline & \multicolumn{2}{|c|}{ Frequency } & \multicolumn{2}{|c|}{ Duration } & \multicolumn{2}{|c|}{ Latency } \\
\hline & $\chi^{2}$ & $p$ & $\chi^{2}$ & $p$ & $\chi^{2}$ & $p$ \\
\hline Sex & 13.4 & 0.00025 & 0.04 & 0.84 & 0.18 & 0.67 \\
\hline Age & 6.1 & 0.014 & 2.03 & 0.15 & 11.2 & 0.00081 \\
\hline Exon1 & 0.13 & 0.71 & 0.39 & 0.53 & 0.75 & 0.39 \\
\hline Exon3 & 0.78 & 0.38 & 3.25 & 0.072 & 0.13 & 0.72 \\
\hline Intron2 & 12.1 & 0.0024 & 9.23 & 0.010 & 6.13 & 0.047 \\
\hline
\end{tabular}


(a)

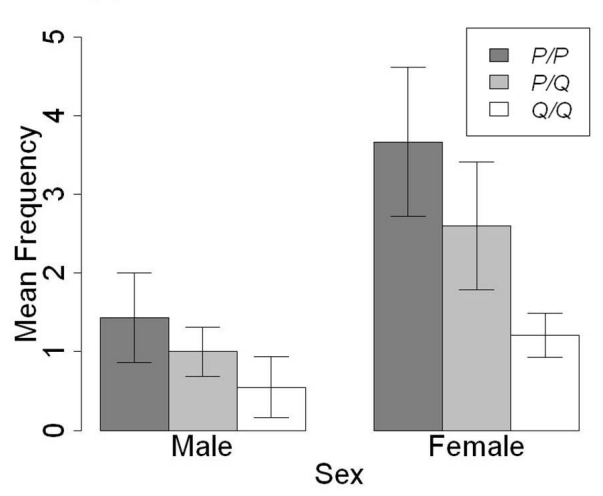

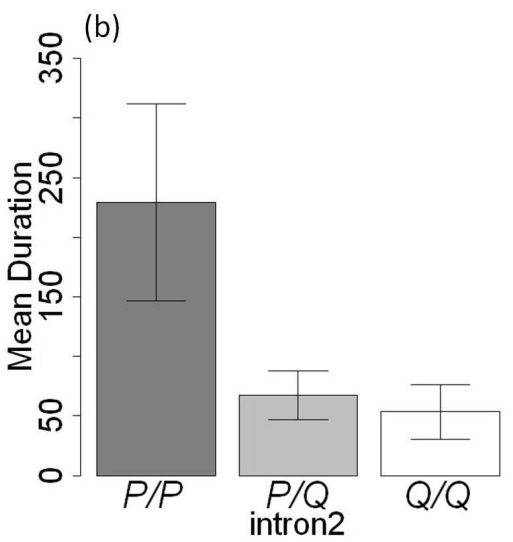

Figure 1. Mean frequency (a), duration (b), and latency (c) of gazing for each genotype group of DRD4 intron2. The frequency result is separated by sex to reflect the significant effect. Duration and latency are represented by number of frames (30 frames/sec). Error bars represent standard error of means (SEM).

Sex, age, and genotype of intron2 were significantly associated with the frequency of gazing behavior. The effects of exon1 and exon3 were not significant. Females looked at their owners more frequently than males $\left(\chi^{2}=\right.$ 13.4, $p=0.00025$, Figure 1(a)). Frequency increased with age $\left(\chi^{2}=6.1, p=0.014\right)$. DRD4 intron2 genotype was significantly associated with frequency $\left(\chi^{2}=12.1, p\right.$ $=0.0024$, Figure 1(a)). Mean frequency was highest in dogs carrying $P / P$ genotype $(P / P: 2.5, P / Q: 1.9, Q / Q$ : $0.9)$.

For duration, a significant effect of $D R D 4$ intron2 was found $\left(\chi^{2}=9.23, p=0.010\right.$, Figure 1(b)). Mean duration was longest in dogs carrying $P / P(P / P: 229.4, P / Q$ : 67.4, $Q / Q$ : 53.4). The effects of other variables were not significant, but there was a tendency for dogs carrying short allele in exon3 to show shorter gazing durations $\left(\chi^{2}=\right.$ $3.25, p=0.072$ ).

For latency, we analyzed only individuals that showed at least one occurrence of gazing ( $N=35,12$ males and 23 females). Significant effects of age and intron2 were found. Latency significantly decreased with age $\chi^{2}=$ $11.2, p=0.00081)$. Intron2 was also significantly associated with latency $\left(\chi^{2}=6.13, p=0.045\right.$, Figure 1(c)). Mean latency was longest in dogs carrying $Q / Q$ genotype (P/P: 571.5, $P / Q:$ 641.7, $Q / Q: 904.5)$. The effects of other variables were not significant.

\section{DISCUSSION}

We analyzed the association between three polymerphic sites in dog DRD4 gene and gazing toward the owner in an experimentally induced difficult situation. We found that the genotype of $D R D 4$ intron2 region was significantly associated with gazing behavior. Dogs carrying $P / P$ genotype looked at their owner the most frequently, for longest, and earliest. The intron2 polymorphism in $\operatorname{dog} D R D 4$ is based on 17 base pairs inser- tion/deletion [10]. This polymorphism was reported to affect the expression of DRD4 and be associated with social impulsivity [13]. These results suggest that intron2 polymorphism in DRD4 may affect social aspects of behavior and cognition in dogs. Previous research showed that dogs carrying shorter allele $(P)$ were less friendly toward a stranger. In the current study, shorter allele appeared to be associated with more owner-dependent traits. Based on these results, dogs carrying shorter allele are predicted to be more dependent on their owners but more discriminating against strangers. This prediction awaits confirmation through additional behavioral tests and questionnaire surveys.

Human-directed gazing was affected by not only the genotype but also sex and age; duration of gazing was longer and latency to gaze was shorter as dogs got older. A previous study also reported that dogs' gazing behavior changed during development [15]. This may reflect the effect of experience; older dogs probably have a longer history of asking for human help. Together, these results suggest that the multivariate model analysis is important because behavioral traits are likely to be affected by many variables.

Our sample consisted of various breeds, which meant that we could not test the interaction between genotypes and breeds. The allele frequency of $D R D 4$ was reported to be different between breeds $[9,10]$. Replication using a larger sample size consisting of a single breed is desirable to evaluate any interaction between breeds and genotypes.

In conclusion, our results suggest that intron2 polymorphism in dog DRD4 may indicate an important marker gene for the study of genetics of dogs' social cognition and behavior. Furthermore, use of an unsolvable task may be valuable for obtaining objective measures of dogs' behavioral traits. 


\section{ACKNOWLEDGEMENTS}

This study was supported financially by the Japan Society for the Promotion of Sciences (JSPS) with Grants-in-aid for Scientific Research (\#21310150 to MI-M and \#20220004 to KF).

\section{REFERENCES}

[1] Miklosi, A., Polgardi, R., Topal, J. and Csanyi, V. (1998) Use of experimenter-given cues in dogs. Animal Cognition, 1, 113-121. doi:10.1007/s100710050016

[2] Gacsi, M., Miklosi, A., Varga, O., Topal, J. and Csanyi, V. (2004) Are readers of our face readers of our minds? Dogs (Canis familiaris) show situation-dependent recognition of human's attention. Animal Cognition, 7, 144153. doi:10.1007/s10071-003-0205-8

[3] Hare, B., Brown, M., Williamson, C. and Tomasello, M. (2002) The domestication of social cognition in dogs. Science, 298, 1634-1636. doi:10.1126/science.1072702

[4] Hare, B. and Tomasello, M. (2005) Human-like social skills in dogs? Trends in Cognitive Sciences, 9, 439-444. doi:10.1016/j.tics.2005.07.003

[5] Miklósi, Á., Kubinyi, E., Topál, J., Gácsi, M., Virányi, Z. and Csányi, V. (2003) A simple reason for a big difference: Wolves do not look back at humans, but dogs do. Current Biology, 13, 763-766. doi:10.1016/S0960-9822(03)00263-X

[6] Inoue-Murayama, M. (2009) Genetic polymorphism as a background of animal behavior. Animal Science Journal, 80, 113-120. doi:10.1111/j.1740-0929.2008.00623.x

[7] Benjamin, J., Li, L., Patterson, C., Greenberg, B.D., Murphy, D.L. and Hamer, D.H. (1996) Population and familial association between the D4 dopamine receptor gene and measures of novelty seeking. Nature Genetics, 12, 81-84. doi:10.1038/ng0196-81

[8] Ebstein, R.P., Novick, O., Roberto Umansky, R., Priel, B., Osher, Y., Blaine, D., Bennett, E.R., Nemanov, L., Katz, M. and Belmaker, R.H. (1996) Dopamine D4 receptor (D4DR) exon III polymorphism associated with the human personality trait of novelty seeking. Nature Genetics, 12, 78-80. doi:10.1038/ng0196-78
[9] Ito, H., Nara, H., Inoue-Murayama, M., Shimada, M.K., Koshimura, A., Ueda, Y., Kitagawa, H., Takeuchi, Y., Mori, Y., Murayama, Y., Morita, M., Iwasaki, T., Ota, K., Tanabe, Y. and Ito, S. (2004) Allele frequency distribution of the canine dopamine receptor D4 gene exon III and I in 23 breeds. Journal of Veterinary Medical Science, 66, 815820. doi:10.1292/jvms.66.815

[10] Nara, H., Inoue-Murayama, M., Koshimura, A., Sugiyama, A., Murayama, Y., Maejima, M., Ueda, Y., Ito, H., Randi, E., Kim, H., Ha, J., Kitagawa, H., Takeuchi, Y., Mori, Y., Iwasaki, T., Morita, M., Ota, K. and Ito, S. (2005) Novel polymorphism of the canine dopamine receptor D4 gene intron II region. Animal Science Journal, 76, 81-86. doi:10.1111/j.1740-0929.2005.00241.x

[11] Niimi, Y., Inoue-Murayama, M., Murayama, Y., Ito, S. and Iwasaki, T. (1999) Allelic variation of the D4 dopamine receptor polymorphic region in two dog breeds, Golden retriever and Shiba. Journal of Veterinary Medical Science, 61, 1281-1286. doi:10.1292/jvms.61.1281

[12] Hejjas, K., Vas, J., Topal, J., Szantai, E., Ronai, Z., Szekely, A., Kubinyi, E., Horvath, Z., Sasvari-Szekely, M. and Miklosi, A. (2007) Association of polymorphisms in the dopamine D4 receptor gene and the activity-impulsivity endophenotype in dogs. Animal Genetics, 38, 629-633. doi:10.1111/j.1365-2052.2007.01657.x

[13] Hejjas, K., Kubinyi, E., Ronai, Z., Szekely, A, Vas, J., Miklósi, A, Sasvari-Szekely, M. and Kereszturi, E. (2009) Molecular and behavioral analysis of the intron 2 repeat polymorphism in the canine dopamine D4 receptor gene. Genes, Brain, and Behavior, 8, 330-336. doi:10.1111/j.1601-183X.2008.00475.X

[14] Koski, S.E. (2011) How to measure animal personality and why does it matter? Integrating the psychological and biological approaches to animal personality. In: InoueMurayama, M., Kawamura, S. and Weiss, A., Eds, From Genes to Animal Behavior, Springer, Berlin, 115-136. doi:10.1007/978-4-431-53892-9_5

[15] Passalacqua, C., Marshall-Pescini, S., Barnard, S., Lakatos, G., Valsecchi, P. and Prato-Previde, E. (2011) Human-directed gazing behavior in puppies and adult dogs, Canis lupus familiaris. Animal Behavior, 82, 1043-1050. doi:10.1016/j.anbehav.2011.07.039 